\title{
SELF-ADJUSTED ACTIVE CONTOURS USING MULTI-DIRECTIONAL TEXTURE CUES
}

\author{
E.A. Mylona, M.A. Savelonas, D. Maroulis \\ Department of Informatics and Telecommunications, University of Athens, Greece \\ \{emylona,msavel,dmaroulis\}@di.uoa.gr
}

\begin{abstract}
Parameterization is an open issue in active contour research, associated with the cumbersome and time-consuming process of empirical adjustment. This work introduces a novel framework for self-adjustment of region-based active contours, based on multi-directional texture cues. The latter are mined by applying filtering transforms characterized by multi-resolution, anisotropy, localization and directionality. This process yields to entropy-based image "heatmaps", used to weight the regularization and data fidelity terms, which guide contour evolution. Experimental evaluation is performed on a large benchmark dataset as well as on textured images. The segmentation results demonstrate that the proposed framework is capable of accelerating contour convergence, maintaining a segmentation quality which is comparable to the one obtained by empirically adjusted active contours.
\end{abstract}

Index Terms-Active Contours, Local Feature Space, Automated Parameter Adjustment, Segmentation.

\section{INTRODUCTION}

Successful active contour applications highly hinge on determining the parameter values involved. In most cases, parameters are empirically adjusted through a cumbersome trial and error process, with technical skills being a prerequisite for the domain user. Although in this way segmentation results can be optimized on a case-by-case basis, they lack objectivity, whereas the parameterization involved cannot be adopted in alternative active contour contexts.

A number of ad hoc approaches have been proposed to cope with the issue of parameterization. In Pluempitiwiriyawej et al. [1] and Tsai et al. [2], parameters are updated within the iterative procedure of active contour evolution. Nonetheless, possible erroneous behavior of the active contour in the early evolution stages has not been encountered. Kokkinos et al. [3] introduced a statistical approach based on the posterior probabilities of texture, edge and intensity cues in a locally adaptive manner.
Nevertheless, their approach requires extensive developer support. Additionally, Keuper et al. [4] and Liu et al. [5] proposed a dynamic parameter adjustment, primarily dependent on the shape of the object of interest. Iakovidis et al. [6] and Hsu et al. [7] presented a framework for optimization of active contour parameters based on genetic algorithms. These heuristic approaches converge slowly in locally optimal solutions. Allili et al. [8] proposed a method for estimating hyper-parameters, which is capable of balancing the contribution of regularization and data fidelity terms. However, manual parameter tuning is still involved. Moreover, Yushkevich et al. [9] developed a toolkit for level-set segmentation of images of anatomical structures. Although their GUI is friendly to non-expert users, parameter settings are still empirically fixed. A precursor of the proposed work has been introduced in [10], where active contour parameters are automatically adjusted based on image entropy, which is derived by the application of the gray-level co-occurrence matrix. Nevertheless, this method cannot account for information residing in multiple scales, whereas the associated computation cost is high.

This work introduces a novel framework for selfadjustment of region-based active contours, based on texture cues. The latter are mined by filtering methods characterized by multi-resolution, anisotropy, localization and directionality. This information is encoded by entropy-based image "heatmaps", which are able to weight the regularization and data fidelity terms appearing in the region-based energy functional. The main idea is to amplify forces which guide contour away from noisy, high-entropy regions and reduce forces imposed within the proximity of structured regions, naturally related to target edges.

The proposed framework is more objective, as well as unsupervised, thus freeing domain users from technical considerations. Moreover, it can be applied to various image modalities, such as biomedical, textured and real-world images and does not require a priori knowledge on the shape of the target region.

The outline of this paper is organized as follows: Section 2 presents the proposed framework. Section 3 demonstrates the experimental results whereas conclusions of this study are summarized in Section 4. 


\section{PROPOSED FRAMEWORK}

Textural information along image edges can be used in order to adjust regularization and data fidelity parameters in an intuitive way. In this light, we use the Contourlet Transform (CT) [12] as a multi-dimensional, multi-scale texture representation method.

\subsection{Entropy Heatmaps}

CT facilitates the identification of edges in multiple directions, providing an inherent noise filtering mechanism, since directionality cannot be attributed to noise. In addition, CT retrieves vital information at multiple scales, whereas it is computationally efficient, by employing iterated directional filter-banks (DFB) which capture point discontinuities by means of the Laplacian Pyramid (LP). In the context of the proposed framework, CT output is used to calculate entropy measures. The derived entropy heatmaps are used to adjust regularization and data fidelity parameters.

The downsampled low-pass and band-pass image versions contain lower and higher frequencies, respectively. The band-pass image contains detailed information of point discontinuities associated with target edges. Furthermore, DFB is implemented by an $l$-level binary tree which leads to $2^{l}$ subbands. In the first stage, a two-channel quincunx filterbank [13] with fan filters divides the 2D spectrum into vertical and horizontal directions. In the second stage, a shearing operator reorders the samples. As a result, different directional frequencies are captured at each decomposition level.

Figure 1 portrays a sample image grid obtained by the Amsterdam Library of Object Images (ALOI) database [14] being fed into CT filter-bank. The sample is decomposed to the finest and second finest scales which are partitioned into four directional subbands. The band-pass directional subbands represent the local feature space. For each subband of image $I_{j k}$, entropy measures are calculated according to:

$$
\begin{gathered}
I E_{j k}=-\sum_{n=1}^{N_{j k}} \sum_{m=1}^{M_{j k}} p_{j k}(m, n) \cdot \log p_{j k}(m, n) \\
p_{j k}(m, n)=\frac{\left|I_{j k}(m, n)\right|^{2}}{\sqrt{\sum_{n=1}^{N_{j k}} \sum_{m=1}^{M_{j k}}\left[I_{j k}(m, n)\right]^{2}}}
\end{gathered}
$$

where $I E_{j k}$ is the information entropy of the $k^{\text {th }}$ direction in the $j^{\text {th }}$ level, $M_{j k}$ is the row size and $N_{j k}$ the column size of the subband image. The maximum entropy value $I E_{j k}$ of the most informative scale $j$, which depends on $N$ and $M$, is calculated and assigned to all pixels of each grid. The result is considered as an entropy image heatmap. The entropy measure is selected as an appropriate indicator to discard a noisy edge. Providing that the structure of an edge region is well ordered or not, each edge is identified as target or noisy, respectively.

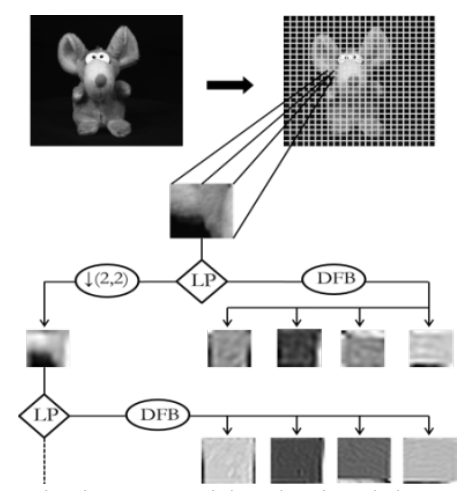

Fig. 1: A sample image grid, obtained by ALOI database [14], is fed into CT filter-bank.

\subsection{Contour Evolution}

Region-based active contours are guided by the minimization of an energy functional $E_{\text {image }}$ :

$E_{\text {image }}=w_{\text {regularization }} \cdot F_{\text {regularization }}+w_{\text {data fidelity }} \cdot F_{\text {data fidelity }}$

where $F_{\text {regularization }}$ and $F_{\text {data fidelity }}$ are associated with regularization and data fidelity forces respectively, whereas $w_{\text {regularization }}$ and $w_{\text {data fidelity }}$ are weighting parameters.

In the context of the proposed framework, the weighting parameters are interconnected, yielding a balance between regularization and data fidelity terms. Weight calculations are based on the entropy heatmaps derived in the previous subsection:

$w_{\text {regularization }} \propto\left(1 / w_{\text {data fidelity }}\right) \times N \times M, w_{\text {data fidelity }}=\arg _{l j k} \max \left(I_{j k}\left(I_{j k}\right)\right)$

These weights: (a) amplify region-based forces in noisy, high-entropy regions, driving the contour away, and (b) reduce region-based forces in structured regions associated with target edges. As a result, iterations dedicated to erroneous local minima are avoided, speeding up contour convergence. Figure 2 illustrates: (a) an artificial bipartite textured image consisting of a target object and the initial contour (green circle), (b) a sketch of data fidelity forces in an iteration of contour evolution, and (c) a sketch of these forces in the next iteration.

\section{RESULTS}

The proposed framework is embedded into two active contour models [15], [16] so as to evaluate the segmentation 


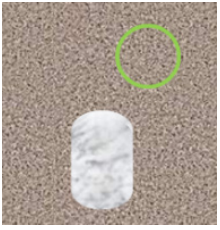

(a)

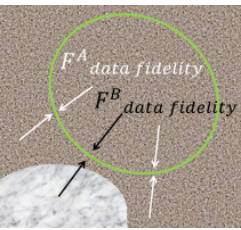

(b)

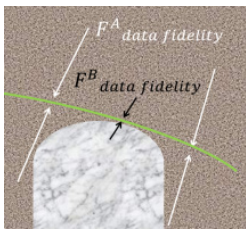

(c)
Fig. 2: (a) Artificial bipartite textured image consisting of a target object and the initial contour (green circle), (b) sketch of data fidelity forces in an iteration of contour evolution, where $F_{\text {data fidelity }}^{A}$ (white arrows) relate to noisy edges and $F_{\text {data fidelity }}^{B}$ (black arrows) reflect structured target edges, (c) sketch of data fidelity forces in the next iteration of contour evolution, where $F_{\text {data fidelity }}^{A}$ is amplified (long white arrows) and $F_{\text {data fidelity }}^{B}$ is reduced (short black arrows).

performance of manual versus self-adjusted version. The Chan-Vese [15] model has been implemented in Matlab, whilst for the model of Bresson et al. [16], the author's Matlab code [17] is used. The multi-scale and multidirectional decomposition stage of $\mathrm{CT}$ is performed by means of the ' $9-7$ ' biorthogonal filter [18]. The original, manually parameterized algorithms were used as baseline, being compared to the self-adjusted versions by means of the region overlap measure, known as the Tannimoto Coefficient (TC) [19],

$T C=\frac{N(A \cap B)}{N(A \cup B)}$

where $A$ is the region delineated by the segmentation method under evaluation, $B$ is the ground truth region and $N()$ indicates the number of pixels of the enclosed region. Experiments were conducted on 8 textured images provided in [17] as well as on 100 images of the ALOI database [14] in order to evaluate the proposed framework on a large benchmark dataset. The ALOI database has been utilized in literature for segmentation purposes [20]-[22]. All test images were captured with varying viewing and illumination angles, resulting in challenging shades. It should be noted that the manual parameterizations used as baseline, follow the authors' suggestions.

Figure 3 illustrates segmentation results of the selfadjusted Chan-Vese model, using the proposed framework. Several images of ALOI database, such as the illustrated 'mouse', 'parrot' and 'jar', contain only intensity-based information, whereas some also contain textured regions, as is the case with 'knot of wire'. Moreover, various images, such as "mouse" and "parrot", consist of difficult shades as well as challenging illuminations, as is the case with "jar". It is evident that in the manual case, contour convergence is delayed. On the contrary, in self-adjustment, forces guiding contour evolution are appropriately amplified in non-target, high-entropy regions, accelerating convergence.

The self-adjusted version achieves an average $T C$ value of $96.9 \pm 1.6 \%$, which is comparable to the $T C$ value obtained by the empirically adjusted version. However, the selfadjusted version converges in 10-20 times less iterations. The empirically adjusted version achieves a $T C$ value of $58.4 \pm 14.3 \%$, with regards to all ALOI images tested, in the same iteration that the self-adjusted version has converged.

Figure 4 illustrates bipartite textured images utilized in [15], whereas Fig. 5 depicts segmentation results of the manual and self-adjusted versions for the model of Bresson et al. It is evident that both versions converge to the actual object boundaries, resulting in comparable segmentation quality. However, it should be considered that with manual adjustment it is always possible to set "optimal" values after laborious, time-consuming experimentation.

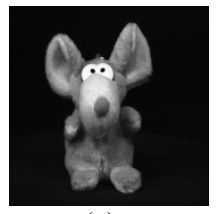

(a)

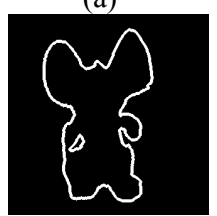

$\left(a_{1}\right)$

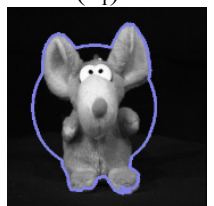

$\left(a_{2}\right)$

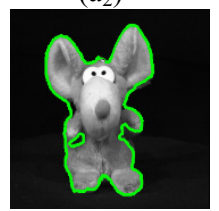

$\left(a_{3}\right)$

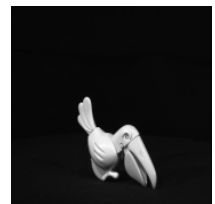

(b)

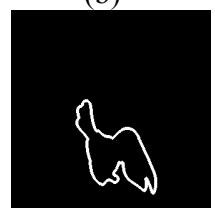

$\left(b_{1}\right)$

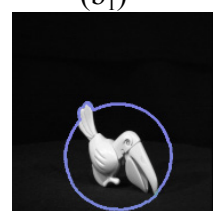

$\left(b_{2}\right)$

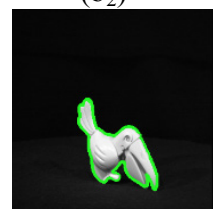

$\left(b_{3}\right)$

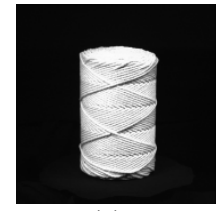

(c)

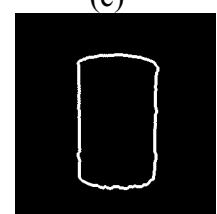

$\left(c_{1}\right)$

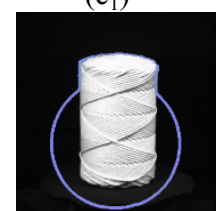

$\left(c_{2}\right)$

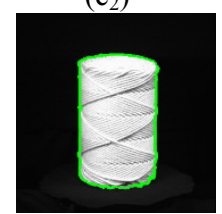

$\left(c_{3}\right)$

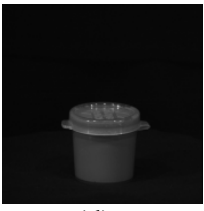

(d)

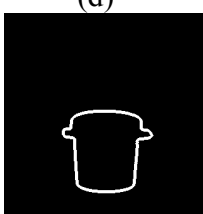

$\left(\mathrm{d}_{1}\right)$

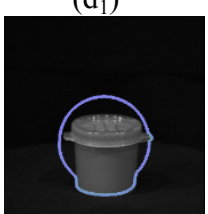

$\left(d_{2}\right)$

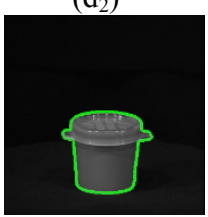

$\left(\mathrm{d}_{3}\right)$
Fig. 3: (a)-(d) Test images obtained by ALOI database, $\left(a_{1}\right)$ $\left(d_{1}\right)$ ground truth images, $\left(a_{2}\right)-\left(d_{2}\right)$ segmentation evolution of the manual version in the same iteration that the selfadjusted version has converged, $\left(a_{3}\right)-\left(d_{3}\right)$ segmentation results of the self-adjusted Chan-Vese model.

\section{CONCLUSIONS}

This work presents a novel framework for self-adjustment of region-based active contours based on multi-directional texture cues. The latter are fed into entropy-based image "heatmaps" which are able to weight forces and guide contour evolution. The proposed framework has been experimentally evaluated on a large benchmark dataset as well as on textured images by comparing the segmentation performance obtained by manual versus self-adjusted version of two state-of-the-art region-based active contour 
models. The experimental results demonstrate that the proposed framework is capable of accelerating contour convergence, whereas it obtains a segmentation quality comparable to the one obtained with manual parameterization.

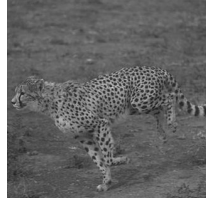

(a)

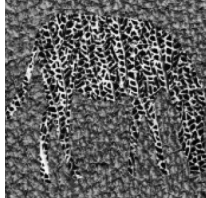

(b)
Fig. 4: (a), (b) Bipartite textured images.

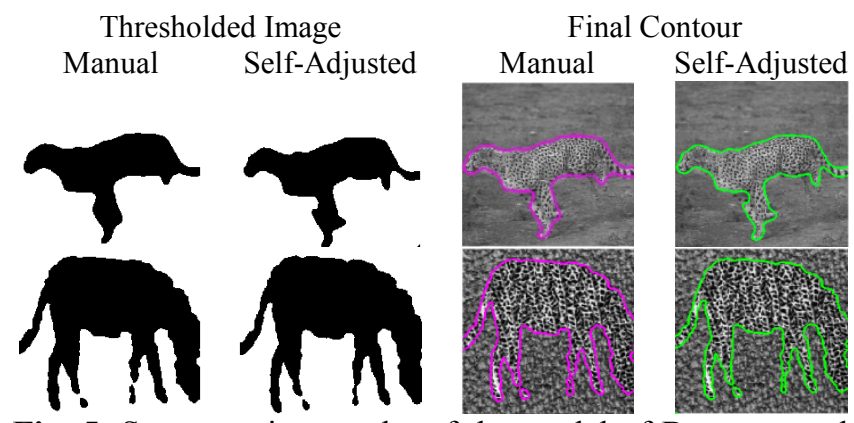

Fig. 5: Segmentation results of the model of Bresson et al. Magenta and green contours correspond to manual and selfadjusted version, respectively.

\section{ACKNOWLEDGEMENTS}

This work was partially supported by the National and Kapodestrian University of Athens, Special Account of Research Grants.

\section{REFERENCES}

[1] C. Pluempitiwiriyawej, J.M.F. Moura, Y.J.L. Wu and C. Ho, "STACS: New Active Contour Scheme for Cardiac MR Image Segmentation," IEEE Transactions on Medical Imaging, vol. 24, no. 5, pp. 593-603, 2005.

[2] A. Tsai, A. Yezzi, W. Wells, C. Tempany, D. Tucker, A. Fan, W.E. Grimson and A. Willsky, "A Shape-Based Approach to the Segmentation of Medical Imagery Using Level Sets," IEEE Transactions on Medical Imaging, vol. 22 no. 2, pp. 137-154, 2003.

[3] I. Kokkinos, G. Evangelopoulos and P. Maragos, "Texture Analysis and Segmentation Using Modulation Features, Generative Models and Weighted Curve Evolution," IEEE Transactions on Pattern Analysis and Machine Intelligence, vol. 31, no. 1, pp. 142-157, 2009.

[4] M. Keuper, T. Schmidt, J. Padeken, P. Heun, K. Palme, H. Burkhardt and O. Ronneberger, "3D Deformable Surfaces with Locally Self-Adjusting Parameters - A Robust Method to Determine Cell Nucleus Shapes," Proc. IEEE International
Conference on Pattern Recognition (ICPR), pp. 2254-2257, 2010.

[5] X. Liu, Y.M. Cheung, M. Li and H. Liu, "A Lip Extraction Method Using Localized Active Contour Model with Automatic Parameter Selection," Proc. IEEE International Conference on Pattern Recognition (ICPR), pp. 4332-4335, 2010.

[6] D. Iakovidis, M. Savelonas, S. Karkanis and D. Maroulis, "A Genetically Optimized Level Set Approach to Segmentation of Thyroid Ultrasound Images," Applied Intelligence, Springer-Verlag, vol. 27, no. 3, pp. 193-203, 2007.

[7] C.Y. Hsu, C.Y. Liu and C.M. Chen, "Automatic Segmentation of Liver PET Images," Computerized Medical Imaging and Graphics, vol. 32, pp. 601-610, 2008.

[8] M. Allili, D. Ziou, “An Approach for Dynamic Combination of Region and Boundary Information in Segmentation,"Proc.International Conference on Pattern Recognition (ICPR), pp. 1-4, 2008.

[9] P.A. Yushkevich, J. Piven, H. Cody, S. Ho, J.C. Gee and G. Gerig, "User-Guided Level Set Segmentation of Anatomical Structures with ITK - SNAP," Insight Journal, Special Issue on ISC/NA-MIC/MICCAI Workshop on Open-Source Software, 2005.

[10] E.A. Mylona, M.A. Savelonas and D. Maroulis, "EntropyBased Spatially-Varying Adjustment of Active Contour Parameters," Proc. IEEE International Conference on Image Processing (ICIP), Orlando, FL, U.S.A., 2012.

[11] E. Nadernejad, "Edge Detection Techniques: Evaluations and Comparisons," Applied Mathematical Sciences, vol. 2, no. 31, pp. 1507-1520, 2008.

[12] M.N. Do and M. Vetterli, "The Contourlet Transform: An Efficient Directional Multiresolution Image Representation," IEEE Transactions on Image Processing, vol. 14, no. 12, pp. 2091-2106, 2005.

[13] M. Vetterli, "Multidimensional Subband Coding: Some Theory and Algorithms," IEEE Transactions on Signal Processing, vol. 6, no. 2, pp. 97-112, 1984.

[14] J.M. Geusebroek, G.J. Burghouts and A.W.M. Smeulders, "The Amsterdam Library of Object Images," International Journal of Computer Vision, vol. 61, no. 1, pp. 103-112, 2005.

[15] T.F. Chan and L.A. Vese, "Active Contours Without Edges," IEEE Transactions on Image Processing, vol. 10, no. 2, pp. 266-277, 2001.

[16] X. Bresson, S. Esedoglu, P. Vandergheynst, J. Thiran and S. Osher, "Fast Global Minimization of the Active Contour/Snake Model," Journal of Mathematical Imaging and Vision, vol. 28, no. 2, pp. 151-167, 2007.

[17] X. Bresson, City University of Hong Kong, 2010, http://www.cs.cityu.edu.hk/ xbresson/.

[18] D.D.-Y. Po and M.N. Do, "Directional Multiscale Modeling of Images using the Contourlet Transform," IEEE Transactions on Image Processing, vol. 15, no. 6, pp. 16101620, 2006.

[19] W.R. Crum, O. Camara and D.L.G. Hill, "Generalized Overlap Measures for Evaluation and Validation in Medical Image Analysis," IEEE Transactions on Medical Imaging, vol. 25, no. 11, pp. 1451-1461, 2006.

[20] I.B. Ayed and A. Mitiche, "A Region Merging Prior for Variational Level Set Image Segmentation," IEEE Transactions on Image Processing, vol. 17, no. 12, pp. 23012311,2008 
[21] L.A. de C. Jorge, E.J. Ferreira, H. de S. Ruiz, V.O. Roda and A. Gonzaga, "Automatic Selection of Color Channels for Segmentation of Aerial Images with Photometric Variations," Proc. IEEE International Conference on Computer Vision (ICCV), pp. 1-7, 2007.

[22] Q. Wu and J. Fang, "Parametric Kernel-Driven Active Contours for Image Segmentation," Journal of Electronic Imaging, vol. 21, no. 4, pp. 043015, 2012. 